\title{
Capacitor Energy Variations in MMC Using Harmonics Injection
}

Research Article

\author{
Kamran Hafeez ${ }^{1 *}$, Shahid A. Khan¹, Alex Van den Bossche², Kamel Djamel Eddinne ${ }^{3}$ \\ ${ }^{1}$ Electrical and Computer Engineering Department, COMSATS University Islamabad, Pakistan \\ ${ }^{2}$ Electrical Energy, Metals, Mechanical Constructions and Systems, Gent University, 9052 Gent, Belgium \\ ${ }^{3}$ Satellite Development Center CDS, P. O. B. 4065, 31000 Ibn Rochd USTO, Oran, Algeria
}

Received August 22, 2020; Accepted August 27, 2020

\begin{abstract}
Modular multilevel converter (MMC) is considered as a good technology for high-voltage direct current (HVDC) transmission systems. It can generate a good quality staircase output voltage waveform due to the cascaded connection of large number of identical sub-modules (SMs). Energy variations in SM capacitor lead to capacitor voltage fluctuations which affect the size and stability of MMC. Traditionally MMC arm voltages are controlled using direct modulation method. The closed loop and open loop compensated modulation methods are implemented to overcome the drawbacks created in directmodulation method. However, these methods rely on the accurate measurements or estimation of SM capacitor voltages. In this paper, capacitor energy variations in MMC based on direct modulation method under three different categories using proportional-resonant (PR) controllers are discussed as (a) pure sinusoidal arm current (b) sinusoidal arm current with addition of large 2 nd harmonic (c) sinusoidal arm current with addition of 2 nd +4 th harmonics. Analytical and simulation results show reduction in capacitor energy variations in $\mathrm{MMC}$ while injecting harmonics in the arm currents using direct modulation method.
\end{abstract}

Keywords: $M M C \bullet H V D C \bullet$ power converter • capacitor energy

\section{Introduction}

Amongst the voltage source converters (VSCs), the MMC is emerging as the most prominent technology for HVDCbased transmission systems due to less switching losses and scalable output voltage (Rohner et al., 2010). The MMC internal dynamics consist of SM voltage fluctuations in capacitors and the circulating current that arises due to the voltage differences between the converter arms and the DC voltage (Li et al., 2015). In direct modulation method, average SM capacitor voltage is assumed, which results in large capacitor energy variations leading to capacitor voltage fluctuations and circulating current in MMCs, whereas in open loop compensated modulation technique, capacitor voltage ripple is estimated to compensate arm voltage oscillations based on the measurement of amplitude and phase of output current. However it involves measurement and transmission delays (Antonopoulos et al., 2009). Capacitor voltage fluctuations can be reduced by injecting 2 nd harmonic in the circulating current reference in a closed loop method. This method also needs accurate information of amplitude and phase of output current (Picas et al., 2012). Similarly 2nd harmonic is injected in the circulating current in a closed loop method based on the information of instantaneous values of power in the arms of MMC (Wang et al., 2019). The injection of 2nd harmonic in a voltage reference using multi-carrier phase shifted PWM is reported in Li et al. (2006). However, it reduces the capacitor voltage fluctuations in MMC based on closed loop modulation method. Multiple harmonics are injected in the circulating current reference to reduce the capacitor voltage fluctuations using a closed loop strategy (Pou et al., 2015). A closed loop MMC leg energy controller is implemented to manage upper and lower arm capacitor energies to reduce SM capacitor voltage fluctuations (Bergna et al., 2013). Alternately, double line frequency circulating current can be suppressed to reduce capacitor voltage fluctuations in MMC using d-q frame 
proportional-integral (PI) controllers (Qingrui et al., 2011). Proportional-resonant and proportional-integral-resonant (PIR) controllers are implemented to control arm currents and suppress the circulating current in MMC (Moon et al., 2015; Ziwen and Shihong, 2019). However, these controllers put further burden on the system. Whereas in this paper a new method is proposed to minimize capacitor energy variations in MMCs. This method adopts a strategy by assuming a pure sinusoidal arm current in a direct modulation method. The $2 \mathrm{nd}+4$ th harmonics are injected in the arm currents of an MMC. This will restrict excessive negative currents in a period resulting in reduction of capacitor energy variations. In this method SM capacitor voltages are neither measured nor estimated compared to closed loop and open loop compensated modulation methods.

To the best of author's knowledge the key contribution of this paper can be described as given below:

(1) Unlike Li et al. (2006) and Pou et al. (2015), who adopt a closed loop strategy to reduce SM capacitor voltage fluctuations, in this paper 2nd and 4th harmonics are added to the arm currents using direct modulation method. The proposed method eliminates the need for a separate controller for the estimation of capacitor voltage ripples.

(2) Unlike Moon et al. (2015) and Ziwen and Shihong (2019), who utilize PR and PIR controllers to suppress the double line frequency circulating current in MMC, in this paper PR controllers are designed to track harmonics added in the arm currents of MMC.

(3) PR controllers are designed to track arm currents in MMC under three different categories: (a) pure sinusoidal arm current at $50 \mathrm{~Hz}$ (b) sinusoidal arm current at $50 \mathrm{~Hz}$ with addition of large 2nd harmonic (c) sinusoidal arm current at $50 \mathrm{~Hz}$ with the addition of $2 \mathrm{nd}+4$ th harmonics.

Less capacitor energy variations are witnessed by injecting harmonics in the arm currents based on direct modulation method in one leg of an MMC, as reported in subsequent section of this paper.

\section{Three-phase MMC}

A balanced three-phase MMC can be represented by considering SMs of each arm as an arm equivalent circuit with variable capacitors as represented by a single phase system shown in Figure 1. The capacitor voltages in each arm can be considered as controlled voltage sources.

The AC side current in phase $a$ shown in Figure 1 can be described as;

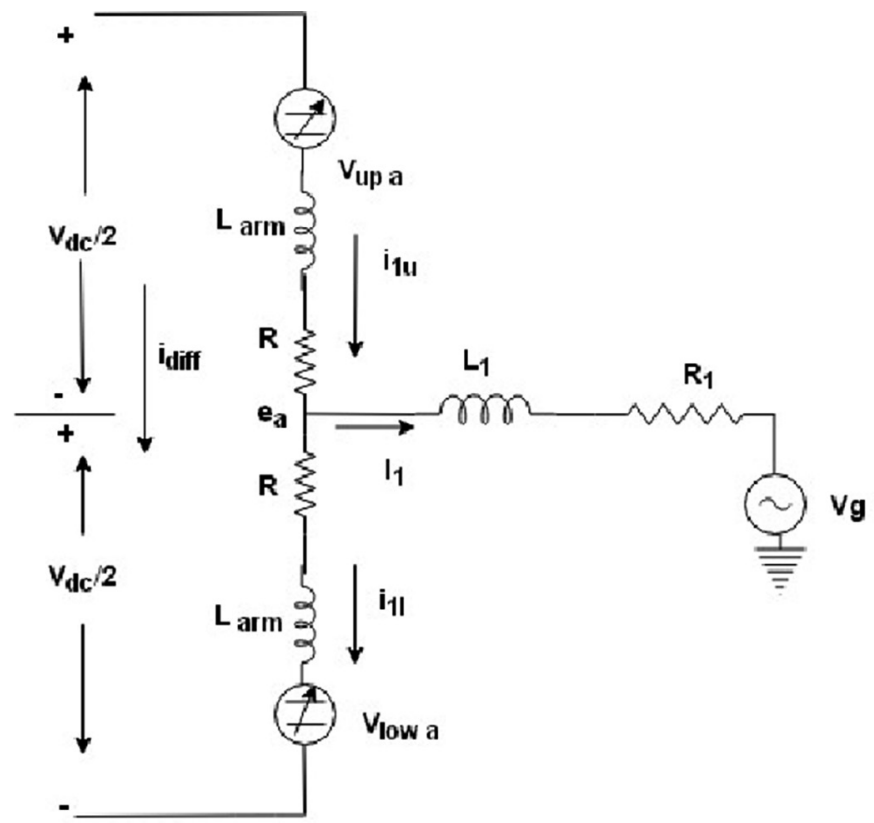

Fig. 1. One leg of three-phase MMC 


$$
i_{1}=i_{1 u}-i_{1 l}
$$

The upper and lower am currents in MMC $i_{1 u}$ and $i_{1 /}$ are given as;

$$
\begin{aligned}
& i_{1 u}=i_{\text {diff }}+\frac{i_{1}}{2} \\
& i_{1 l}=i_{\text {diff }}-\frac{i_{1}}{2}
\end{aligned}
$$

where $i_{1}$ is the output current of MMC and $i_{\text {diff }}$ is the differential current comprising two components i.e. a DC component $i_{\text {diff_dc }}$ and a negative sequence double line frequency alternating current component $i_{\text {diff_ac }}$ or circulating current. Now using equations (2) and (3) the upper and lower currents in each arm are equal to half of the phase current $i_{1}$, one-third of DC current $i_{d c}$, plus the circulating current $i_{c i r}$.

$$
\begin{aligned}
& i_{1 u}=\frac{i_{1}}{2}+\frac{i_{d c}}{3}+i_{c i r} \\
& i_{1 l}=-\frac{i_{1}}{2}+\frac{i_{d c}}{3}+i_{c i r}
\end{aligned}
$$

Unlike Angquist et al. (2011), who adopt an open loop compensation method which assumes $i_{\text {diff }}$ as a pure DC component. Furthermore, It estimates capacitor energy stored in MMC arms based on the measurement of output current and phase angle. In this paper, $i_{\text {diff }}$ is considered as a pure alternating current component. The dynamics of MMC using average model using equations (4) and (5) can be formulated as:

$$
\begin{aligned}
& i_{1 u}=\frac{c^{a r m} d V_{u(t)}^{\Sigma}}{n_{u} d t} \\
& i_{1 l}=\frac{c^{a r m} d V_{l(t)}^{\Sigma}}{n_{l} d t}
\end{aligned}
$$

where $c^{\text {arm }}$ is the arm capacitance, $V_{u}^{\sum}, V_{l}^{\sum}$ represent arm capacitor voltages and $V_{\text {upa }}, V_{\text {lowa }}$ represent the arm voltages

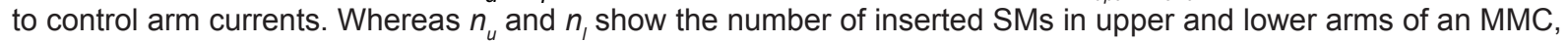
which becomes a continuous parameter between 0 and 1 (Ahmed et al., 2014). In this dynamic average model, switching pattern is neglected with even voltage distribution among the SMs of an MMC.

Different types of MMC modulation methods are shown in Figure 2. The phase disposition (PD), alternate phase disposition (APOD) and phase opposition disposition (POD) waveforms produce large circulating current inside MMC. Phase shifted (PS-PWM) method produces less harmonics due to the cancellation of harmonics as multi-carrier waveforms are shifted symmetrically according to the number of SMs inside the MMC; however

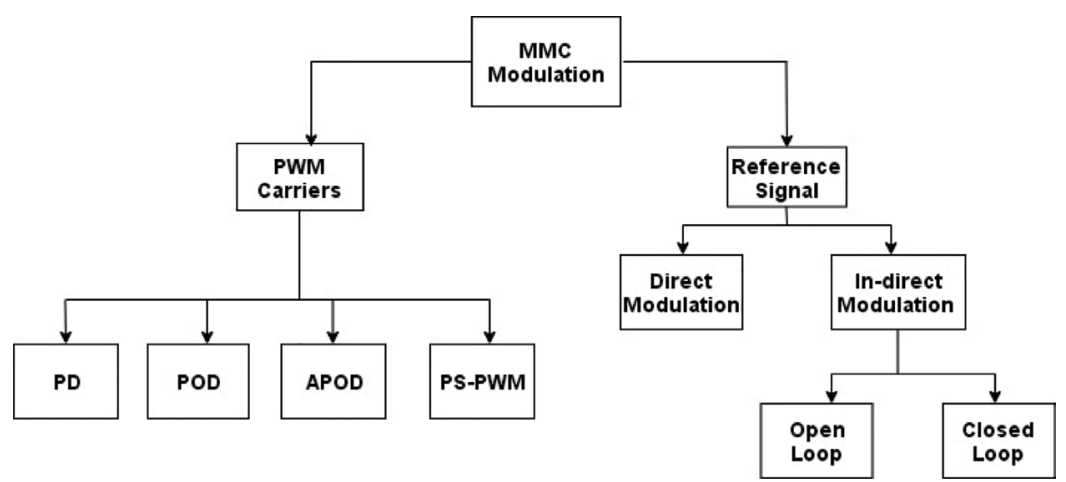

Fig. 2. $M M C$ modulation methods 
it needs a separate controller to balance the capacitor voltages. Two reference signals are generated for upper and lower arms of the MMC to control arm voltages in direct modulation method. Similarly for indirect modulation, two reference signals are generated; however, in this method capacitor voltages are measured or estimated in a closed loop or open loop fashion involving transmission and measurement delays (Debnath et al., 2015). Therefore, in this paper, direct modulation method is used without involving measurements of capacitor voltages.

\section{Capacitor energy variations using harmonics injection}

Change in capacitor energy over half a period $(T=0.02 \mathrm{~s})$ with a sinusoidal arm current, sinusoidal arm current + large 2 nd harmonic and sinusoidal arm current $+2 \mathrm{nd}+4$ th harmonics can be calculated using Figure 1 . Whereas the value of $t$ is between -0.01 and $0.01 \mathrm{~s}$.

$$
w_{c(t)}=\int_{-0.01}^{t} i_{\operatorname{leg}(t)} \cdot v_{\operatorname{leg}(t)} d t
$$

where leg current and leg voltage can be described as:

$$
\begin{aligned}
& i_{\operatorname{leg}(t)}=I_{a c} \cdot \cos (\omega \cdot t)+I_{d c} \\
& v_{\operatorname{leg}(t)}=V_{d c}-V_{a c}
\end{aligned}
$$

Here, $i_{\text {leg(t) }}$ is the leg current in a converter and $v_{\text {leg(t) }}$ is the leg voltage. Whereas $I_{a c}$ is the amplitude of AC current and $V_{a c}$ is the AC voltage.

Sinusoidal currents in the arm

a) The sinusoidal arm current can be expressed as:

$$
i_{l e g(t)}=I_{a c} \cdot \cos (\omega \cdot t)+I_{d c}
$$

b) Sinusoidal currents in the arm + large 2nd harmonic

The sinusoidal currents in the arm with a large 2 nd harmonic can be expressed as:

$$
i_{l e g(t)}=I_{a c} \cdot \cos (\omega \cdot t)+0.91 \cdot \cos (2 \cdot \omega \cdot t)+I_{d c}
$$

c) Sinusoidal arm current $+2 n d+4$ th harmonics

Adding 2nd and 4th harmonics in equation (6), sinusoidal arm current $+2 \mathrm{nd}+4$ th harmonic can be expressed as:

$$
i_{l e g(t)}=I_{a c} \cdot \cos (\omega \cdot t)+A \cdot \cos (2 \cdot \omega \cdot t)+B \cdot \cos (4 \cdot \omega \cdot t)+I_{d c}
$$

Symbolically differentiating equation (13), the following expressions are derived:

$$
\begin{aligned}
& i_{\operatorname{leg}(t)}=-\omega \cdot \sin (\omega \cdot t)-2 \cdot A \cdot \omega \cdot \sin (2 \cdot \omega \cdot t)-4 \cdot B \cdot \omega \cdot \sin (4 \cdot \omega \cdot t) \\
& i_{\operatorname{leg}(t)}=-\omega^{2} \cdot \cos (\omega \cdot t)-4 \cdot A \cdot \omega^{2} \cdot \cos (2 \cdot \omega \cdot t)-16 \cdot B \cdot \omega^{2} \cdot \cos (4 \cdot \omega \cdot t) \\
& i_{\operatorname{leg}(t)}=\omega^{3} \cdot \sin (\omega \cdot t)+8 \cdot A \cdot \omega^{3} \cdot \sin (2 \cdot \omega \cdot t)+64 \cdot B \cdot \omega^{3} \cdot \sin (4 \cdot \omega \cdot t) \\
& i_{\operatorname{leg}(t)}=-\omega^{4} \cdot \cos (\omega \cdot t)+16 \cdot A \cdot \omega^{4} \cdot \cos (2 \cdot \omega \cdot t)+256 \cdot B \cdot \omega^{4} \cdot \cos (4 \cdot \omega \cdot t)
\end{aligned}
$$

Solving equation (17);

$$
-1+16 . A+256 \cdot B=0
$$

The leg current can be extracted from equation (18) using values $A=\frac{20}{64}$ and $B=\frac{-1}{64}$ given as; 


$$
i_{\operatorname{leg}(t)=} I_{a c}\left[\cos (\omega \cdot t)+\frac{20}{64} \cdot \cos (2 \cdot \omega \cdot t)+\frac{-1}{64} \cdot \cos (4 \cdot \omega \cdot t)\right]+I_{d c}
$$

The analytical results of equation (11), (12) and (19) are plotted in Figure 3. It is evident from these plots that in sinusoidal arm current without considering any harmonics, there is almost no flat bottom current as shown in Figure 3(a). Sinusoidal arm current + large 2 nd harmonic has reduced flat bottom, whereas sinusoidal arm current $+2 n d+4$ th harmonics has more flat bottom current as evident from Figure 3(c), which is believed to be due to the constant current in a part of a period for $120^{\circ}$ confirming less capacitor energy variations in MMC.

\section{Controller design}

Conventionally MMC is controlled using AC-side current controller and a separate circulating current suppression controller (Qin and Saeedifard, 2012). In this paper AC-side current reference is calculated according to the active and reactive power references; more details are given in Moon et al. (2015). However, unlike Moon et al. (2015), arm current references are calculated using AC-side grid current and AC current component of $i_{\text {diff }}$ The proposed arm current control method based on PR controllers is shown in Figure 4(a). The difference of the reference $\left(i_{1 u}^{*}, i_{11}^{*}\right)$ and measured $\left(i_{14}, i_{11}\right)$ arm currents is fed to the PR controllers to generate upper and lower arm voltage references $V_{\text {up a }}^{*}$ and $V_{\text {low a. }}^{*}$. This will control MMC output voltage and current $i_{1}$. The insertion indices for MMC using direct modulation method can be described in equations (20) and (21) (K. Ilves et al., 2012).

$$
n_{u}=\frac{1}{2}[1+\dot{m} \cos (\omega t+\phi)]
$$

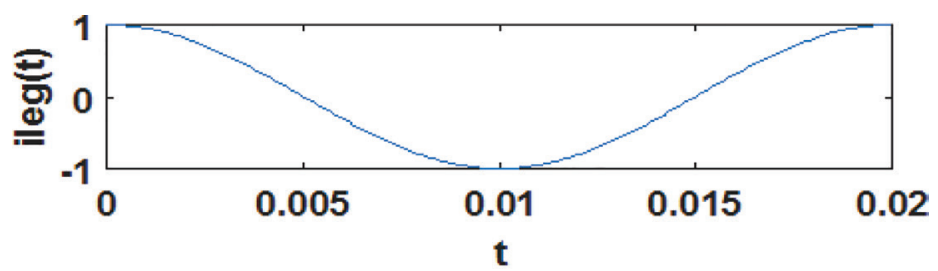

(a)

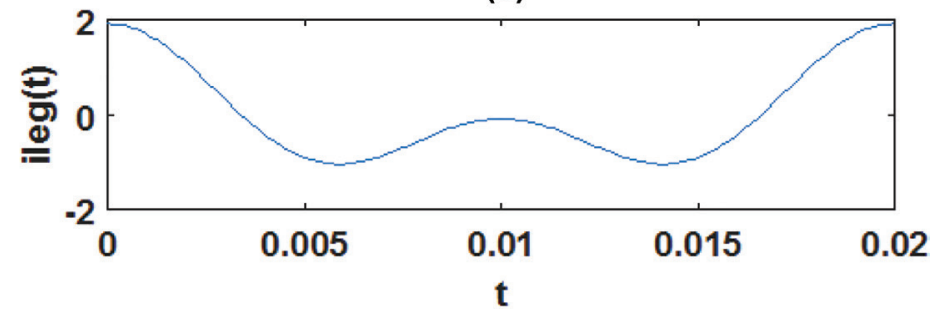

(b)

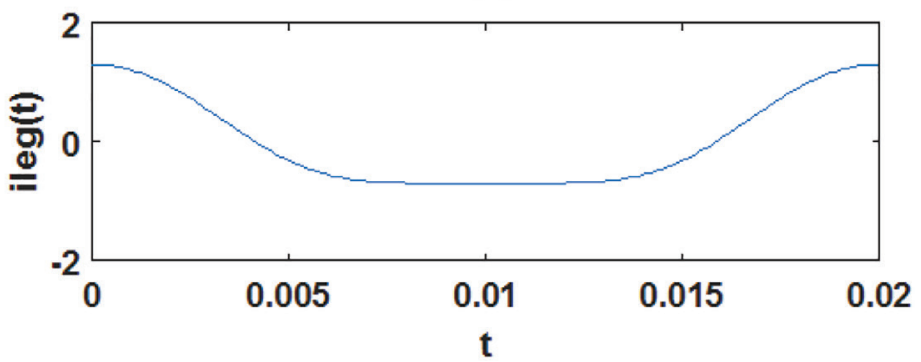

(c)

Fig. 3. Analytical results: (a) sine wave (b) sine wave + large 2 nd harmonic (c) sine wave $+2 n d+4$ th harmonics 

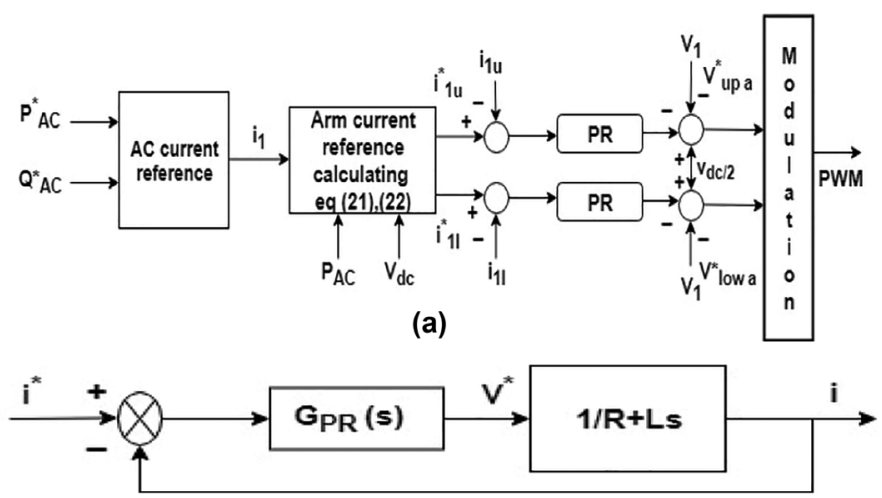

(b)

Fig. 4. (a) Proposed arm current control using PR (b) simplified arm current control loop

$$
n_{l}=\frac{1}{2}[1-\dot{m} \cos (\omega t+\phi)]
$$

where $\dot{m} \in[01]$ is the modulation index, $\phi$ is the phase angle and $\omega$ is the grid frequency.

The simplified form of arm current control loop is shown in Figure 4(b). The output of the PR controller is the reference voltage $v^{*}$ to be applied across the inductor $L_{\text {arm }}$. Whereas $i$ represents the output current measured through the inductor while $i^{*}$ denotes the reference current.

The sinusoidal current reference $i^{\star}$ for upper and lower arms in MMC can be calculated as

$$
\begin{aligned}
& \mathrm{i}^{*}(t)=i_{\text {diff_ac }}+\frac{i_{1}}{2} \\
& \mathrm{i}^{*}(t)=i_{\text {diff_ac }}-\frac{\mathrm{i}_{1}}{2}
\end{aligned}
$$

The MMC arm currents references are calculated based on equations (12), (19), (22) and (23) given as

$$
\begin{aligned}
& i^{*}(t)=i_{\text {diff } a c}+\frac{\mathrm{i}_{1}}{2}+0.91 \cdot \cos (2 \cdot \omega \cdot t) \\
& i^{*}(t)=i_{\text {diff }-a c}+\frac{\mathrm{i}_{1}}{2}+\frac{20}{64} \cdot \cos (2 \cdot \omega \cdot t)+\frac{-1}{64} \cdot \cos (4 \cdot \omega \cdot t)
\end{aligned}
$$

The open loop system transfer function of the plant using Figure 4(b) can be described in equation (26) and (27).

$$
\begin{aligned}
G_{T F \text { sys }}(s) & =\frac{1}{s L+R} \\
G_{T F \text { sys }}(s) & =\frac{1}{0.8+0.076 s}
\end{aligned}
$$

The ideal PR controller is designed to track arm current references given as

$$
G_{P R}=\left(k_{P}+\frac{2 k_{r} s}{s^{2}+\omega_{r}^{2}}\right)
$$

where $k_{p}$ and $k_{r}$ are the proportional and resonant gains and $\omega_{r}$ is the resonant frequency.

The bode plots of ideal PR current controller developed in MATLAB simulation software based on equation (28) are shown in Figure 5. The magnitude of bode plot with $k_{p}=1, k_{r}=1000$ having resonant frequency 


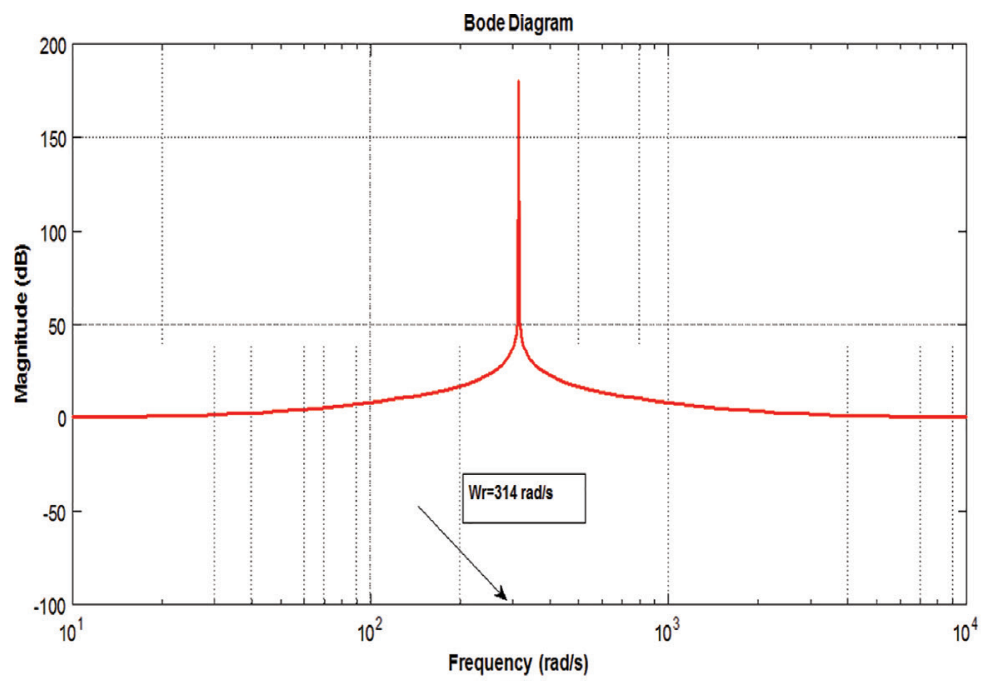

(a)

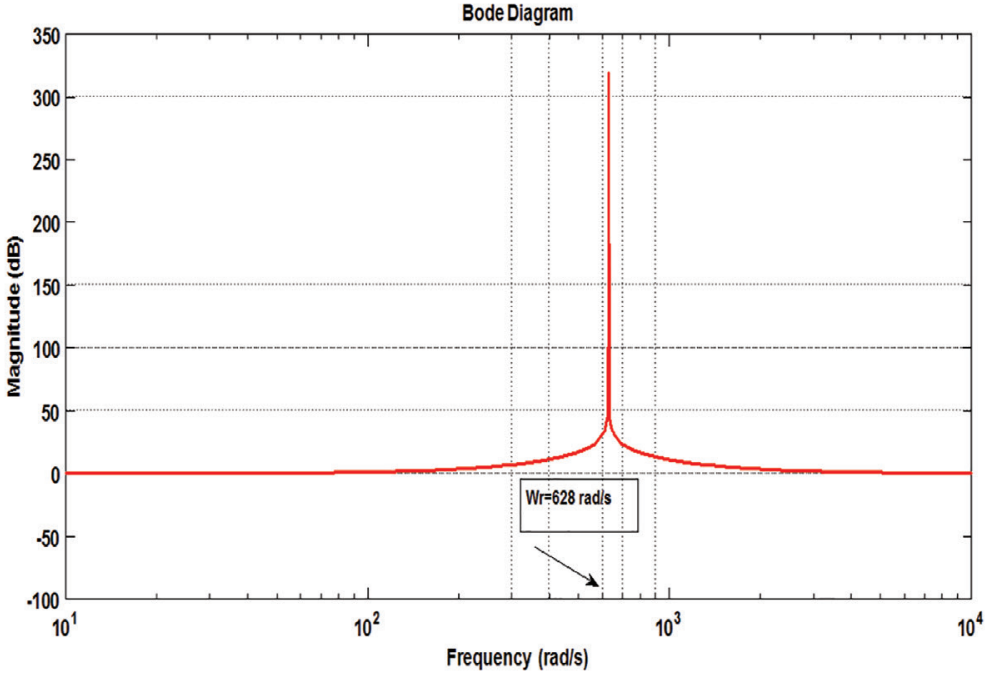

(b)

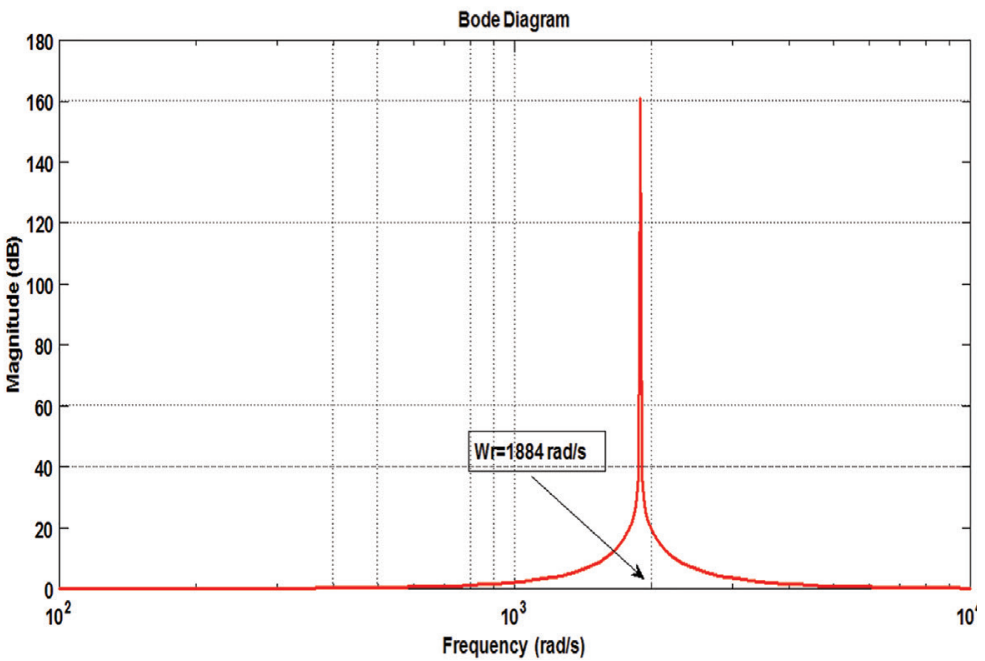

(c)

Fig. 5. Bode plot of ideal PR controller: (a) sinusoidal arm current (b) sinusoidal arm current + large 2 nd harmonic (c) sinusoidal arm current + 2nd +4 th harmonics 
$314 \mathrm{rad} / \mathrm{s}$ to track pure sinusoidal current at $50 \mathrm{~Hz}$ frequency is shown in Figure 5(a). Similarly magnitude of the bode plot of an ideal PR controller with resonant frequency of $628 \mathrm{rad} / \mathrm{s}$ in order to track sinusoidal current at $50 \mathrm{~Hz}$ frequency with addition of large 2nd harmonic is shown in Figure 5(b). Finally, magnitude of bode plot of ideal PR controller with resonant frequency of $1884 \mathrm{rad} / \mathrm{s}$ to track sinusoidal current at $50 \mathrm{~Hz}$ frequency with addition of $2 \mathrm{nd}+4$ th harmonics is shown in Figure $5(\mathrm{c})$. It is evident from these bode plots that there is sufficiently high gain at $\omega_{r}$ frequency. This will result in zero steady state error, which is good for the tracking ability of PR controllers. However, to reduce high gain, a non-ideal PR controller can be implemented as given in equation (29) (Sun et al., 2010):

$$
G_{P R}=\left(k_{p}+\frac{2 k_{r} \omega_{c} s}{s^{2}+2 \omega_{c}^{2}+\omega_{r}^{2}}\right)
$$

where $\omega_{c}$ is the cut-off frequency. The addition of $\omega_{c}$ enables current controller to be less sensitive during frequency changes. The value of $\omega_{c}$ is selected around $12 \mathrm{rad} / \mathrm{s}$.

The current control loop from Figure $4(\mathrm{~b})$ is given as

$$
I(s)=H(s) I^{*}(s)
$$

where $H(s)$ represents the transfer functions of the PR controller and the plant. Bode plots of the current control loop are shown in Figure 6 . The positive gain and phase margins show that the system is stable.

\section{Simulation results}

The parameters of one leg MMC are given in Table 1. The simulation results for all the above discussed cases using data given in Table 1 is shown in Figure 7.

Mathcad software is used to get the simulation results by implementing arm current control strategy shown in Figure 4. Multiplying current with voltage in a leg gives the change in energy in MMC. The MMC model implemented in this paper assumes infinite switching frequency. Therefore, a simple PWM is used based on direct modulation method that only considers fundamental switching frequency, without considering switching harmonics in the converter.

The sinusoidal arm current generated due to direct modulation method without harmonic addition has capacitor energy variations between $\pm 283 \times 1 \times 10^{6} \mathrm{~J}$, which leads to more capacitor voltage fluctuations as shown in Figure 7(a). The sinusoidal arm current with a large 2nd harmonic has least capacitor energy variations, between $\pm 283 \times 4 \times 10^{5} \mathrm{~J}$ as shown in Figure 7(b). However, it contains two maxima and one minima in a period that results in high peak current. Change in capacitor energy in sinusoidal arm current with 2 nd +4 th harmonic is also lower i.e. between $\pm 283 \times 6 \times 10^{5} \mathrm{~J}$ compared to sinusoidal arm current having an additional advantage of reduction in peak current, which is around $7.5 \times 10^{5} \mathrm{~A}$ as compared to $1 \times 10^{6} \mathrm{~A}$ as evident from Figure 7 (b) and Figure $7(\mathrm{c})$. The $w_{c(t)}$ is divided by 283 as a scaling factor to compare it to the peak AC value of voltage and current.

\section{Conclusion}

Capacitor energy variations in MMC with different harmonics injections using PR current controllers are discussed in this paper. These harmonics are injected in the arm currents of MMC using direct modulation methods. PR controllers are designed to control arm currents accurately. Analytical and simulation results show that the lowest change in energy storage in the capacitors might be with a very large 2nd harmonic but at the expense of higher peak current in MMC. The $2 \mathrm{nd}+4$ th harmonics is believed to be a good method to produce less capacitor energy variations. This will lead to reduce capacitor voltage fluctuations with a moderate peak current in MMC. The proposed method will be tested with different MMC models in future work. 


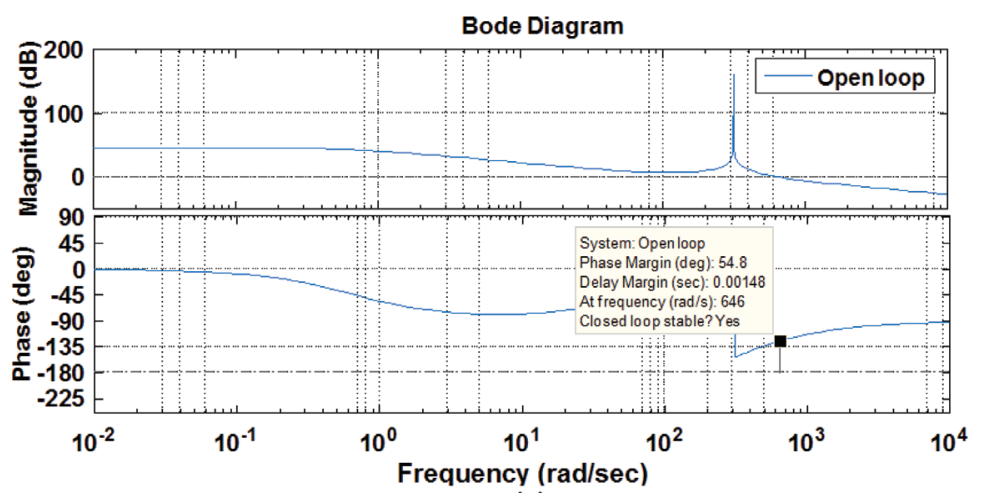

(a)

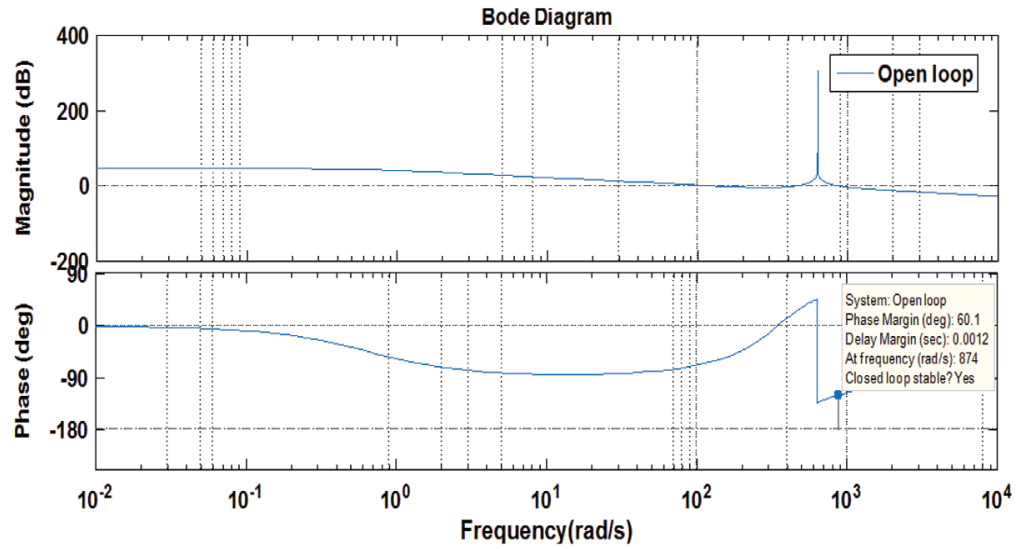

(b)

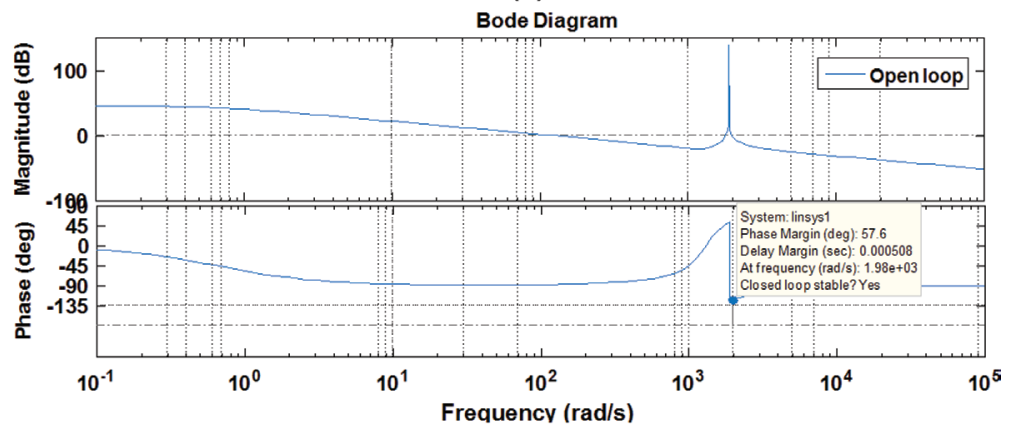

(c)

Fig. 6. Bode plot of current control loop: (a) sinusoidal arm current (b) sinusoidal arm current + large 2 nd harmonic (c) sinusoidal arm current + 2nd +4 th harmonics

Table 1. MMC parameters

\begin{tabular}{lccc}
\hline Quantity & Symbol & Value & Units \\
\hline \hline AC voltage & Vac & 320,000 & $\mathrm{~V}$ \\
DC voltage & Vdc & 330,000 & $\mathrm{~V}$ \\
AC current & lac & 400,000 & $\mathrm{~A}$ \\
DC current & Idc & $1.939 \times 10^{5}$ & $\mathrm{~A}$ \\
Frequency & $f$ & 50 & $\mathrm{~Hz}$ \\
Inductance & $\mathrm{L}$ & 76 & $\mathrm{mH}$ \\
Resistance & $R$ & 0.8 & $\Omega$ \\
\hline
\end{tabular}




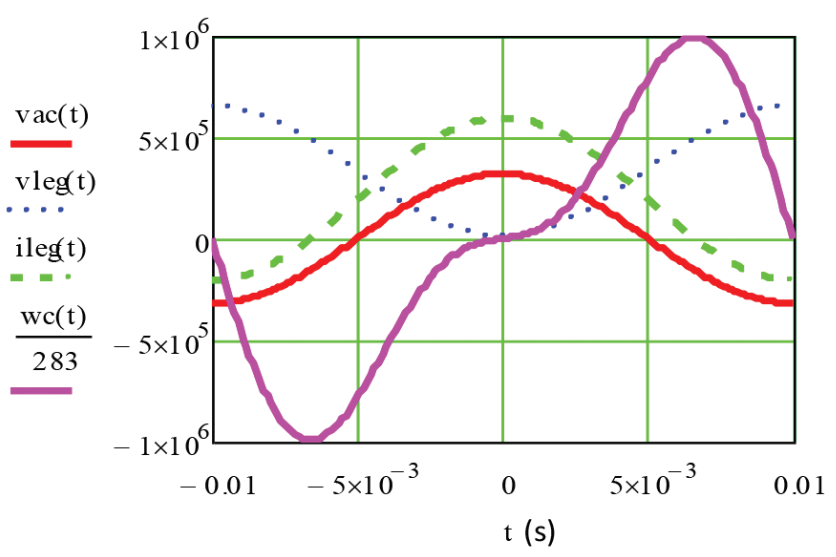

(a)

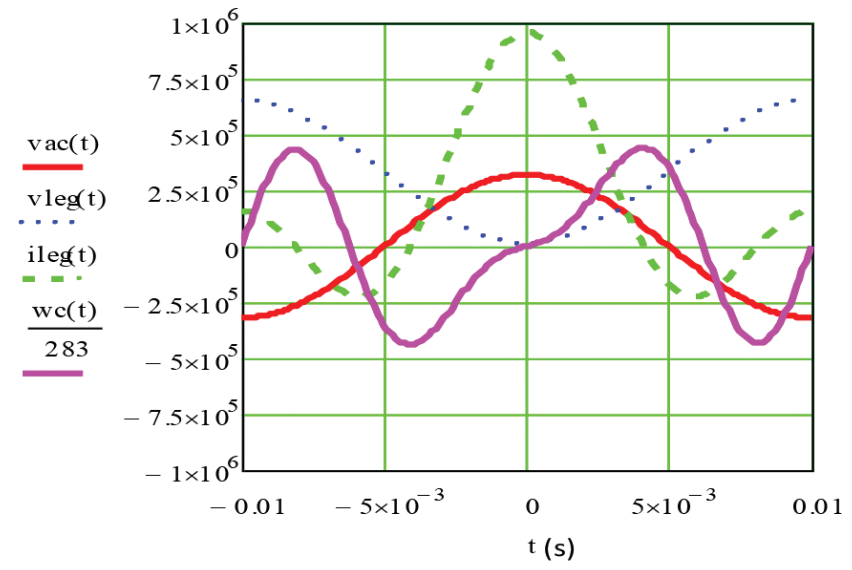

(b)

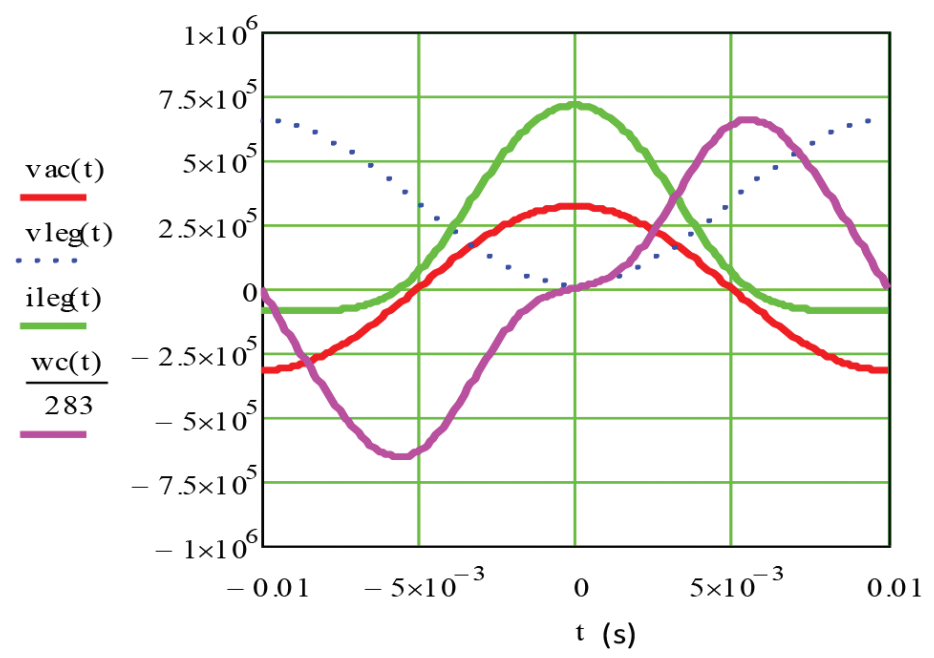

(c)

Fig. 7. Capacitor energy variations in one leg of an MMC: (a) sinusoidal current in the arm (b) sinusoidal current in the arm + large 2 nd harmonic (c) sinusoidal current in the arm $+2 n d+4$ th harmonics 


\section{References}

Ahmed, N., Angquist, L., Norrga, S., Antonopoulos, A., Harnefors, L. and Nee, H. P. (2014). Acomputationally efficient continuous model for the modular multilevel converter. IEEE Journal of Emerging and Selected Topics in Power Electronics, 2, pp. 1139-1148.

Angquist, L., Antonopoulos, A., Siemaszko, D., Ilves, K., Vasiladiotis, M. and Nee, H.-P. (2011). Open-loop control of modular multilevel converters using estimation of stored energy. IEEE Transactions on Industry Applications, 47(6), pp. 2516-2524.

Antonopoulos, A., Angquist, L. and Nee, H. P. (2009). On dynamics and voltage control of the modular multilevel converter. In: Proceeding of the 13th European Conference on Power Electronics and Applications, Barcelona, Spain, pp. 1-10.

Bergna, G., Berne, E., Egrot, P., Lefranc, P., Amir, A., Vannier, J. and Molinas, M. (2013). An energybased controller for HVDC modular multi level converter in decoupled double synchronous reference frame for voltage oscillations reduction. IEEE Transactions on Industrial Electronics, 60, 2360-2371.

Debnath, S., Qin, J., Bahrani, B., Saeedifard, M. and Barbosa, P. (2015). Operation, control, and applications of the modular multilevel converter: A review. IEEE Transactions on Power Electronics, 30(1), pp. 37-53.

Ilves, K., Antonopoulos, A., Norrga, S. and Nee, H. (2012). Steady-state analysis of interaction between harmonic components of arm and line quantities of modular multilevel converters. IEEE Transactions on Power Electronics, 27(1), pp. 57-68.

Li, S., Wang, X., Yao, Z., Li, T. and Peng, Z (2015). Circulating current suppressing strategy for MMCHVDC based on, on ideal proportional resonant controllers under unbalanced grid conditions. IEEE Transactions on Power Electronics, 30, pp. 387-397.

Li, X., Song, Q., Liu, W., Xu, S., Zhu, Z. and Li, X. (2006). Performance analysis and optimization of circulating current control for modular multilevel converter. IEEE Transactions on Industrial Electronics, 63(2), pp. 716-727.

Moon, J. W., Park, J.-W., Kang, D.-W. and Kim, J.-M. (2015). A control method of HVDC-modular multilevel converter based on arm current under the unbalanced voltage condition. IEEE Transactions on Power Delivery, 30(2), pp. 529-536.

Picas, R., Pou, J., Ceballos, S., Agelidis, V. G. and Saeedifard, M. (2012). Minimization of the capacitor voltage fluctuations of a modular multilevel converter by circulating current control. In: Proceedings of IEEE 38th Annual Conference on IEEE Industrial Electronics Society, Montreal, QC, Canada, pp. 4985-4991.

Pou, J., Ceballos, S., Konstantinou, G., Agelidis, V. G., Picas, R. and Zaragoza, J. (2015). Circulating current injection methods based on instantaneous information for the modular multilevel converter. IEEE Transactions on Industrial Electronics, 62(2), pp. 777-788.

Qin, J. and Saeedifard, M. (2012). Predictive control of a modular multilevel converter for a back-toback HVDC System. IEEE Transactions on Power Delivery, 27(3), pp. 1538-1547.

Qingrui, T., Zheng, X. and Lie, X. (2011). Reduced switching-frequency modulation and circulating current suppression for modular multilevel converters. IEEE Transactions on Power Delivery, 26, pp. 2009-2017.

Rohner, S., Bernet, S., Hiller, M. and Sommer, R. (2010). Modulation, losses, and semiconductor requirements of modular multilevel converters. IEEE Transactions on Industrial Electronics, 57(8), pp. 2633-2642.

Sun, J., Chen, B., Guo, S., Zha, X., Xiong, L. and Gong, J. (2010). High-reliability and harmonicsharing dual parallel topology for active power filter. In: Proceedings of IEEE Power Electronics for Distributed Generation Systems, Hefei, China, 16-18, pp. 240-245.

Wang, J., Han, X., Ma, H. and Bai, Z. (2019). Analysis and injection control of circulating current for modular multilevel converters. IEEE Transactions on Industrial Electronics, 66(3), pp. 2280-2290.

Ziwen, L., Shihong, M., Zhihua, F., Yilong, K., and Qingyu, T. (2019). Analysis of the performance characteristics and arm current control for modular multilevel converter with asymmetric arm parameters. International Journal of Electrical Power \& Energy Systems, 110, pp. 258-270. 